\title{
Layered Approach for Predicting Protein Subcellular Localization in Yeast Microarray Data
}

\author{
A. Kumaravel ${ }^{1 *}$ and R. Pradeepa ${ }^{2}$ \\ 'Professor and Dean, Department of Computer Science and Engineering, Bharath University, Selaiyur, \\ Chennai-600073, India; drkumaravel@gmail.com \\ ${ }^{2}$ PG Student, Department of Computer Science and Engineering, Bharath University, Selaiyur, Chennai - 600073; \\ pradimca@gmail.com
}

\begin{abstract}
Subcellular localization is a well-designed representation of proteins. We need a fully automatic and reliable prediction system for protein subcellular localization, especially for the analysis of large-scale of yeast microarray data. In this paper we consider the dataset with multi classes and propose the classification for each location of protein subcellular in a separate layer. In this work, a multi-classification approach for subcellular localization is designed and developed to achieve high efficiency and improve the prediction and classification accuracy. The rule based Ripper method has been found to predict the subcellular localization of proteins from their protein microarray data, compared to other classifiers.
\end{abstract}

Keywords: Data Mining, Microarray, Classification, Layered Approach, Protein Subcellular Localization.

\section{Introduction}

Genome function annotation including the assignment of a function for a potential gene in the raw sequence is now the hot topic in bioinformatics. Subcellular localization is a key functional characteristic of potential gene product such as proteins. Therefore, a fully automatic and reliable prediction system for protein subcellular localization would be very useful. Numerous stabs have been made to predict protein subcellular localization. Maximum of these prediction method scan be classified into two categories: one is based on the recognition of protein $\mathrm{N}$-terminal sorting signals and the other is based on amino acid composition [1]. Recently, they proposed an integrated prediction system for subcellular localization using neural networks based on individual sorting signal predictions or Support Vector Mechanism for general purpose supervised pattern recognition. This paper introduces a new prediction method for protein subcellular localization based on yeast dataset. Classification is perhaps the most familiar and most popular data mining technique. Prediction can be thought of as

\footnotetext{
* Corresponding author:
}

A. Kumaravel (drkumaravel@gmail.com) classifying an attribute value into one of a set of possible classes. Here, we construct a prediction system for subcellular localization called Ripper based on the Data mining classification method. The results show that the prediction accuracy is significantly improved with this novel method and the method is very robust to errors in the yeast microarray data [2].

\section{Problem Statement}

Protein subcellular localization prediction is a multi-class classification problem. Here, the class number is equal to 10. A simple strategy to handle the multi-class classification is to reduce the multi-classification to a series of binary classifications. For a k-class classification, k Layers are constructed. The $i^{\text {th }}$ layer will be trained with all of the samples in the $\mathrm{i}^{\text {th }}$ class with positive labels and all other samples with negative labels. We refer to this way as one-versusrest. At Last one unknown sample is classified into class 
that corresponds to the one-versus-rest with the highest output value.

\subsection{The Proposed Layered-model Subcellular Localization}

Our system has the capability of classifying micro array data. The data is input, which identifies if this record is in particular location or rest. If the record is identified as specified location then the module would raise a flag to the administrator that the coming record is a specified location which is taken into account and the other all locations are taken together, which consists of ten sequential Layers, one for each class type (ERL, NUC, POX, MIT, ME1, ME2, ME3, CYT, EXC, \& VAC) [3]. Each Layer is responsible for identifying the location of coming record according to its class type. Else the locations are taken together as rest. Then each layer act as a filters that classifies the location of each layer category which eliminate the need of further processing at subsequent layers but we took in consideration the propagation of errors as to simulate the real system and results be more accurate and real [4].

In many situations, there is a trade-off between efficiency and accuracy of the system and there can be various avenues to improve system performance. We implement the Layered Approach to improve overall system performance as our layered model using JRipRule achieves high efficiency and improves the detection and classification with high rate of accuracy. Figure 1 shows the proposed layered model of a subcellular localization.

\section{Experimental Analysis and Results}

In this section, first we collect the Microarray dataset. We apply the dataset in Wekatool [5] to find Classification results. The datasets for these experiments are from Institute of Molecular and Cellular Biology [6].

\subsection{Dataset}

\subsubsection{Dataset Description}

The database of Protein Localization is considered as our experimental dataset. It contains 10 different classes. Number of Instances in this database is 1484 for the
3.1.2 Attribute Description

\begin{tabular}{|c|c|}
\hline Attribute name & Description \\
\hline Sequence Name & $\begin{array}{l}\text { SWISS-PROT database accession } \\
\text { number }\end{array}$ \\
\hline $\mathrm{mcg}$ & $\begin{array}{l}\text { Signal sequence recognition McGeoch's } \\
\text { method. }\end{array}$ \\
\hline gvh & $\begin{array}{l}\text { Signal sequence recognition von Heijne's } \\
\text { method. }\end{array}$ \\
\hline alm & $\begin{array}{l}\text { ALOM membrane spanning region } \\
\text { prediction score. }\end{array}$ \\
\hline mit & $\begin{array}{l}\text { Discriminant analysis of the amino acid } \\
\text { content of the } \mathrm{N} \text {-terminal region score. }\end{array}$ \\
\hline erl & $\begin{array}{l}\text { Presence of "HDEL" substring binary } \\
\text { attribute. }\end{array}$ \\
\hline pox & $\begin{array}{l}\text { Targeting Peroxisomal signal in the } \\
\text { C-terminus. }\end{array}$ \\
\hline vac & $\begin{array}{l}\text { Discriminant analyses of the amino acid } \\
\text { content of vacuolar and extracellular } \\
\text { proteins score. }\end{array}$ \\
\hline nuc & $\begin{array}{l}\text { Discriminant analyses of nuclear } \\
\text { localization signals of nuclear and non- } \\
\text { nuclear proteins score. }\end{array}$ \\
\hline
\end{tabular}

yeast dataset and number of Attributes are 9 (8 predictive, 1 name).The class is the localization site shown in Figure 2.

\subsection{Performance Evaluation}

During the prediction of protein subcellular localization we observe two main challenging issues in this system. First, the number of locations in the subcellular is typically a very small fraction of the total microarray. Therefore the essential step is to select attributes of the various Layers. Second, the locations are classified in their impression and hence, it becomes necessary to treat them differently.

To improve the prediction rate, while maintaining a reasonable overall prediction rate. We have proposed a layered model with various classifiers [7] (BayesNet, NavieBayes, Decision Table and rules Jrip) on values. In layered model we define ten layers that correspond to the ten location groups i.e. NUC layer for predicting NUC location, MIT layer for predicting Probe location, CYT layer for predicting CYT location, EXC for EXC location and so on.

A confusion matrix illustrates the accuracy of the solution to a classification problem [8]. The accuracy and error rates are calculated from confusion matrix [9],

$$
\begin{aligned}
& \text { Accuracy }=(\mathrm{TP}+\mathrm{TN}) /(\mathrm{TP}+\mathrm{FN}+\mathrm{FP}+\mathrm{TN}) * 100 \\
& \text { Error rate }=(\mathrm{FN}+\mathrm{FP}) /(\mathrm{TP}+\mathrm{FN}+\mathrm{FP}+\mathrm{TN}) * 100
\end{aligned}
$$




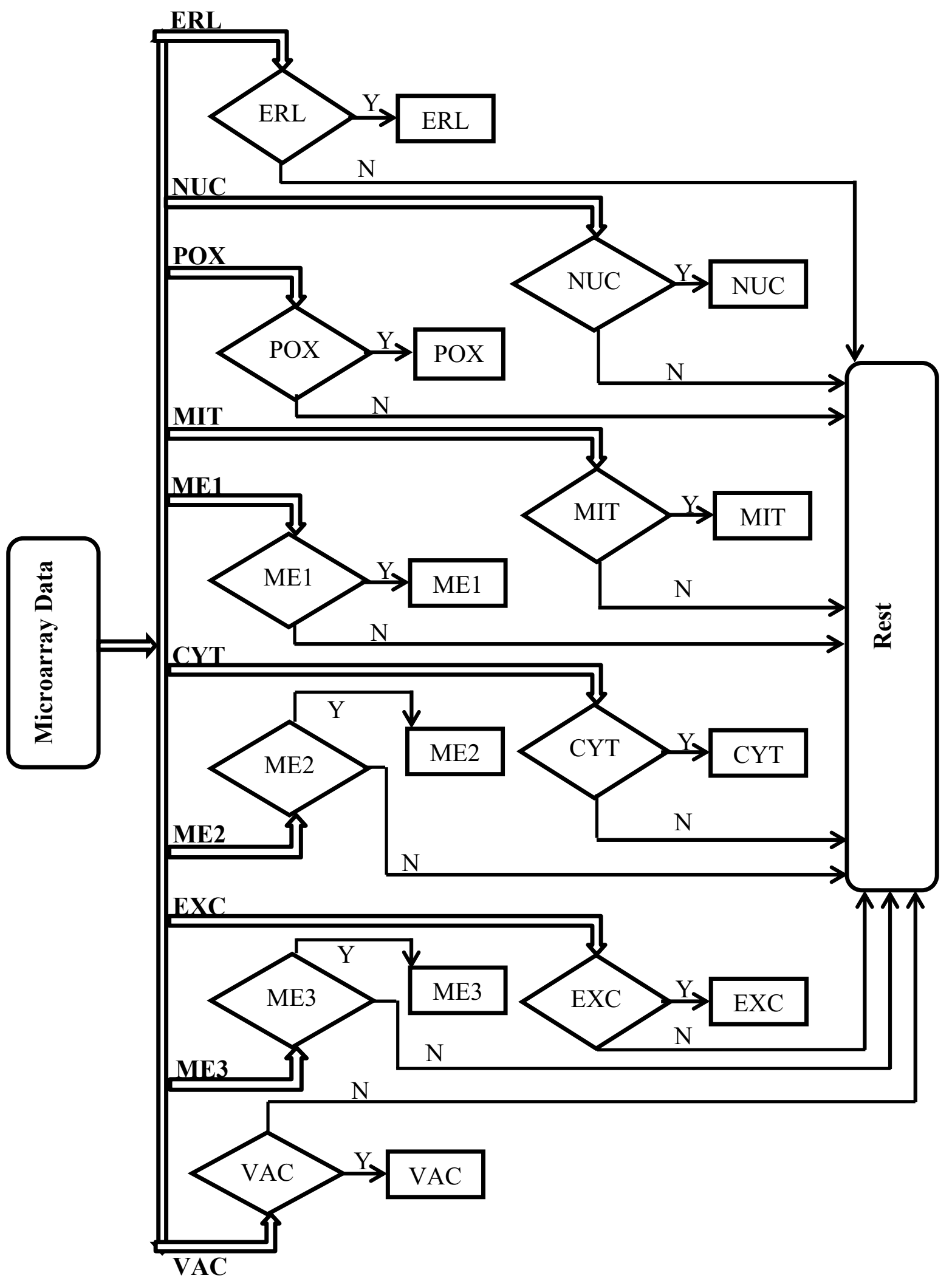

Figure 1. Layered-Model Approach System. 


\begin{tabular}{lcclcc} 
Column 1 & Rest & EXC & Column 1 & Rest & CYT \\
\hline Rest & 1441 & 8 & Rest & 1013 & 8 \\
EXC & 20 & 15 & CYT & 26 & 437 \\
Error Rate & & $1.89 \%$ & Error Rate & & $27.90 \%$ \\
\hline & & & & & \\
Column 1 & Rest & MIT & Column 1 & Rest & NUC \\
\hline Rest & 99 & 145 & Rest & 996 & 59 \\
MIT & 1161 & 79 & NUC & 36 & 393 \\
Error Rate & & $12.00 \%$ & Error Rate & & $6.40 \%$ \\
\hline
\end{tabular}

Table 1. Classification of NUC layer

\begin{tabular}{lcc}
\hline Method & $\begin{array}{c}\text { Correctly } \\
\text { classified }\end{array}$ & $\begin{array}{c}\text { Incorrectly } \\
\text { classified }\end{array}$ \\
\hline bayes.BayesNet & $93.60 \%$ & $6.40 \%$ \\
bayes.NaiveBayes & $88.07 \%$ & $11.93 \%$ \\
rules.Jrip & $79.31 \%$ & $20.69 \%$ \\
rules.Decisiontable & $77.76 \%$ & $22.24 \%$ \\
\hline & & \\
Table 2. Classification of MIT layer & \\
\hline Method & Correctly & Incorrectly \\
& classified & classified \\
\hline bayes.BayesNet & $87.00 \%$ & $13.00 \%$ \\
bayes.NaiveBayes & $88.00 \%$ & $12.00 \%$ \\
rules.Jrip & $87.00 \%$ & $13.00 \%$ \\
rules.Decisiontable & $86.65 \%$ & $13.35 \%$ \\
\hline
\end{tabular}

Table 3. Classification of CYT Layer

\begin{tabular}{llc}
\hline Method & $\begin{array}{c}\text { Correctly } \\
\text { classified }\end{array}$ & $\begin{array}{c}\text { Incorrectly } \\
\text { classified }\end{array}$ \\
\hline bayes.BayesNet & $66.44 \%$ & $33.56 \%$ \\
bayes.NaiveBayes & $68.26 \%$ & $31.74 \%$ \\
rules.Jrip & $72.10 \%$ & $27.90 \%$ \\
rules.Decisiontable & $69.07 \%$ & $30.93 \%$ \\
\hline
\end{tabular}

Positive, FN=False Negative

\subsubsection{Classification of Layers}

Records are classified [11] as locations by classifying the ten sequential layers; coming location to one of the ten classes (ERL, NUC, POX, MIT, ME1, ME2, ME3, CYT, EXC, \& VAC) and identifying its type.

\section{NUC Layer:}

Table 4. Classification of EXC layer

\begin{tabular}{lcc}
\hline Method & $\begin{array}{c}\text { Correctly } \\
\text { classified }\end{array}$ & $\begin{array}{l}\text { Incorrectly } \\
\text { classified }\end{array}$ \\
\hline bayes.BayesNet & $94.95 \%$ & $5.05 \%$ \\
bayes.NaiveBayes & $95.08 \%$ & $4.92 \%$ \\
rules.Jrip & $98.11 \%$ & $1.89 \%$ \\
rules.Decisiontable & $97.50 \%$ & $2.50 \%$ \\
\hline
\end{tabular}

The results of NUC Layer are shown in Table 1.

\section{MIT Layer:}

\section{EXC Layer:}

The results of EXC Layer are shown in Table 4.

The results of MIT Layer are shown in Table 2.

The results of all Layers with best methods are shown in Table 5.

\section{CYT Layer:}

The results of CYT Layer are shown in Table 3.

\section{Performance Comparison with}


Table 5. Layers with best classification method

\begin{tabular}{lccl}
\hline Layer & $\begin{array}{c}\text { Correctly } \\
\text { classified }\end{array}$ & $\begin{array}{c}\text { Incorrectly } \\
\text { classified }\end{array}$ & Best method \\
\hline MIT & $88.00 \%$ & $12.00 \%$ & NAVIBAYES \\
NUC & $93.60 \%$ & $6.40 \%$ & BAYES \\
CYT & $72.10 \%$ & $27.90 \%$ & JRIP \\
ME1 & $98.18 \%$ & $1.89 \%$ & JRIP \\
ME2 & $96.56 \%$ & $3.44 \%$ & JRIP \\
ME3 & $95.28 \%$ & $4.72 \%$ & NAVIBAYES \\
EXC & $98.11 \%$ & $1.89 \%$ & JRIP \\
ERL & $99.93 \%$ & $0.07 \%$ & JRIP \\
POX & $99.13 \%$ & $0.87 \%$ & JRIP \\
VAC & $97.98 \%$ & $2.02 \%$ & JRIP \\
\hline
\end{tabular}

Table 6. Performance comparison with existing system

\begin{tabular}{lcccc}
\hline Methods & $\begin{array}{c}\text { Neural } \\
\text { network }\end{array}$ & $\begin{array}{c}\text { Markov } \\
\text { model }\end{array}$ & SVM & $\begin{array}{c}\text { Proposed } \\
\text { method }\end{array}$ \\
\hline $\begin{array}{l}\text { Accuracy } \\
(\%)\end{array}$ & $\begin{array}{c}\text { Accuracy } \\
(\%)\end{array}$ & $\begin{array}{c}\text { Accuracy } \\
(\%)\end{array}$ & $\begin{array}{c}\text { Accuracy } \\
(\%)\end{array}$ \\
$\begin{array}{l}\text { Cytoplasmic } \\
\text { (CYT) }\end{array}$ & 55 & 78.1 & 76.9 & 72.1 \\
$\begin{array}{l}\text { Extracellular } \\
\text { (EXC) }\end{array}$ & 75 & 62.2 & 80 & 98.1 \\
$\begin{array}{l}\text { Mitochondrial } \\
\text { (MIT) }\end{array}$ & 61 & 69.2 & 56.7 & 88 \\
$\begin{array}{l}\text { Nuclear } \\
\text { (NUC) }\end{array}$ & 72 & 74.1 & 87.4 & 93.6 \\
\hline
\end{tabular}

\section{Existing Approaches}

In this section, we compare the performance of Ripper method predictions were compared with other prediction methods in this field ${ }^{1}$. This information is shown in Table 6.

According to the above table, the accuracy for Extra Cellular (EXC) sequences reached $98.1 \%$ with the rule based JRip method which is much higher than for the other methods.

\section{Conclusions}

A multi-Layer localization prediction system has been developed to achieve high efficiency and improve prediction and classification rate accuracy. The proposed system consists of two stages. First stage is for location detection and the second stage is for location classification. The data is input in the first Stage which identifies if this record is a specified record or rest.

Experimental results specify that the proposed layered model with JRip classifier can result in better prediction of minority classes without hurting the prediction performance of the majority class.

\section{Acknowledgements}

The authors would like to thank the management of Bharath University for the support and encouragement for this research work.

\section{References}

1. Hua S, and Sun Z (2001). Support vector machine approach forprotein subcellularlocalization prediction, Bioinformatics, vol 17(8), 721-728, Available From: http://bioinformatics. oxfordjournals.org/

2. Available From: http://videocast.nih.gov/pdf/rm/Snyder.pdf

3. Gifty P, and Ravichandran J M et al. (2012). Efficient classifier for R2L and U2R attacks, International Journal of Computer Applications, vol 45, No. 21, 0975-8887.

4. Kumaravel A, and Niraisha M (2013). Multi-classification approach for detecting network attacks ICT545, 2013IEEE Conference on Information and Communication Technologies.

5. Available From: Weka: http://www.cs.waikato.ac.nz/ ml/ weka/

6. Protein localization dataset from Institute of Molecular and Cellular Biology Osaka, University, Available From: http:// www.imcb.osaka-u.ac.jp/nakai/psort.html

7. Gaur A, and Richariya V (2011). A layered approach for intrusion detection using meta-modeling with classification techniques, International Journal of Computer Technology and Electronics Engineering (IJCTEE), vol 1(2), 161-168.

8. Kumaravel A, and Pradeepa R (2013). Efficient molecule reduction for drug design by intelligent search methods, International Journal of Pharama and Bio Sciences, vol 4(2): (B), 1023-1029, Available From: http://www.ijpbs. net/cms/php/upload/2348_pdf.pdf

9. Available From: http://webdocs.cs.ualberta.ca/ eisner/measures.html 